\title{
El signo de la hermana María José, un hallazgo semiológico que no se debe olvidar. Reporte de un caso (con video)
}

\section{The Sister Mary Joseph Nodule, a Semiological Finding that Should not Be Forgotten. Case Report with Video}

\author{
Martín Alonso Gómez, ${ }^{1}$ Javier Humberto Riveros, ${ }^{2}$ Hernando Marulanda Fernández. ${ }^{2}$
}

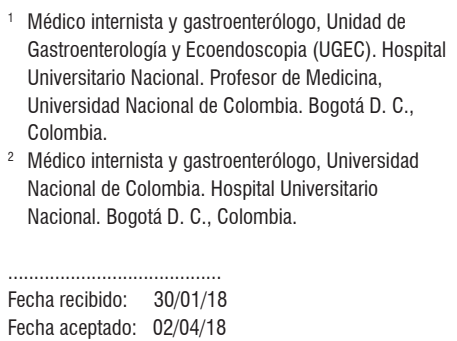

2 Médico internista y gastroenterólogo, Universidad Nacional de Colombia. Hospital Universitario Nacional. Bogotá D. C., Colombia.

Fecha recibido: $\quad 30 / 01 / 18$ Fecha aceptado: $02 / 04 / 18$

\author{
Resumen \\ Se presenta el caso de una paciente con hemorragia digestiva alta, en quien durante el examen físico se \\ documentó el signo de la hermana María José, que se confirmó ulteriormente mediante endoscopia digestiva \\ y estudios histopatológicos la presencia de un cáncer gástrico avanzado. \\ Palabras clave \\ Pólipo, válvula de bola, obstrucción intestinal, polipectomía.
}

\section{Abstract}

We present the case of a patient with upper gastrointestinal bleeding for whom the Sister Mary Joseph nodule was documented during the physical examination. Later, advanced gastric cancer was confirmed by means of digestive endoscopy and histopathological studies.

Keywords

Polyp, ball valve, intestinal obstruction, polypectomy.

\section{INTRODUCCIÓN}

El signo de la hermana María José se refiere a un nódulo maligno en la región periumbilical asociado con una neoplasia abdominal avanzada (1). Dicha denominación se realiza en honor a una religiosa de la congregación de Nuestra Señora de Lourdes, quien trabajó como asistente del cirujano Dr. William Mayo, quien frecuentemente encontraba dicha alteración durante el lavado de la pared abdominal de los pacientes que eran llevados a cirugía por patologías tumorales intraabdominales (2). Su importancia radica en que este puede ser el único signo de presentación, progresión o recurrencia de una patología tumoral (3).

\section{CASO CLÍNICO}

Se trata de una mujer de 48 años, quien consultó a urgencias por dolor abdominal de predominio en el epigastrio, pérdida inexplicada de peso, melenas y hematemesis.

En el examen físico presentó ascitis y un nódulo de aspecto violáceo de $3 \mathrm{~cm}$ y centro necrótico en la región periumbilical (Figura 1).

Dentro de los paraclínicos se encontró anemia microcítica e hipocrómica. La función renal, electrolitos y glucemia eran normales. El perfil hepático presentaba hipoalbuminemia con aminotransferasas normales.

En la endoscopia de vías digestivas altas (EVDA) se encontró una lesión de aspecto tumoral ulcerada, con san- 


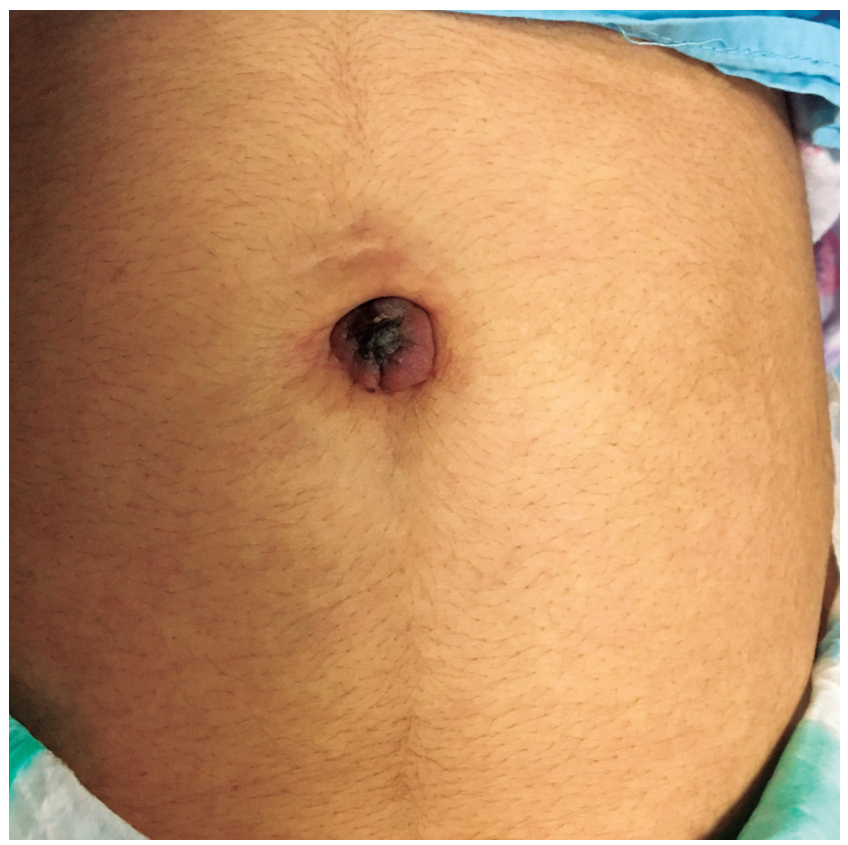

Figura 1. Signo de la hermana María José.

grado espontáneo, bordes irregulares, de aproximadamente $45 \mathrm{~mm}$, localizada hacia la unión corporoantral y extendiéndose hacia la pared posterior asociada con múltiples lesiones satélites de aspecto polipoide (Figura 2, Video 1).

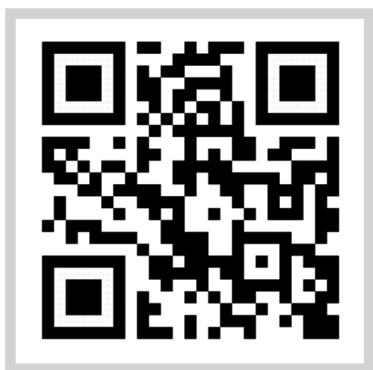

Escanee el código con su smartphone

Video 1. En el video se observa metástasis al ombligo de una paciente con cáncer gástrico avanzado, se puede apreciar una gran masa con sangrado activo. https://youtu.be/K9fyLHGhqL0

La biopsia de la lesión umbilical fue sugestiva de un adenocarcinoma; las muestras tomadas durante la endoscopia confirmaron un adenocarcinoma gástrico moderadamente diferenciado.

\section{DISCUSIÓN}

El signo o nódulo de la hermana María José se refiere a un nódulo en el ombligo de aspecto violáceo y centro necrótico (4). Está relacionado con lesiones metastásicas

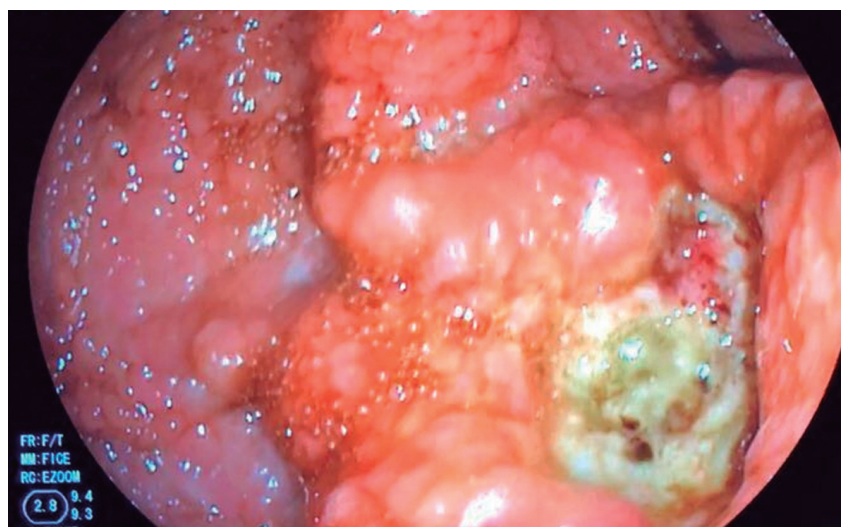

Figura 2. Cáncer gástrico.

de tumores malignos de origen pélvico o intraabdominales (5). Las neoplasias gastrointestinales son su principal causa (6). El mecanismo por el cual el cáncer se propaga a la región periumbilical es desconocido (7), la hipótesis más aceptada es la diseminación directa transperitoneal (8). Dada su localización, es fácilmente accesible y permite el análisis histológico mediante toma de biopsias y, de esta manera, establecer su etiología maligna (8).

El nódulo de la hermana María José está asociado con un pobre pronóstico oncológico y vital (9), y también con estadio avanzado de la enfermedad, múltiples lesiones metastásicas y compromiso peritoneal (10).

\section{REFERENCIAS}

1. Galvañ VG. Sister Mary Joseph's nodule. Ann Intern Med. $1998 ; 128(5): 410$.

2. Urbano F. Sister Joseph's Nodule. Hospital Physician. 2001;44:33-5.

3. Albano EA, Kanter J. Images in clinical medicine. Sister Mary Joseph's nodule. N Engl J Med. 2005;352(18):1913. doi: 10.1056/NEJMicm040708.

4. Powell FC, Cooper AJ, Massa MC, Goellner JR, Su WP. Sister Mary Joseph's nodule: a clinical and histologic study. J Am Acad Dermatol. 1984;10(4):610-5.

5. Majmudar B, Wiskind AK, Croft BN, Dudley AG. The Sister (Mary) Joseph nodule: its significance in gynecology. Gynecol Oncol. 1991;40(2):152-9.

6. Gabriele R, Borghese M, Conte M, Basso L. Sister Mary Joseph's nodule as a first sign of cancer of the cecum: report of a case. Dis Colon Rectum. 2004;47(1):115-7. doi: 10.1007/s10350-003-0018-5.

7. Fill S, Taran A, Schulz HU, Kahl S, Kalinski T, Smith B, et al. Sister Mary Joseph's nodule as the first sign of pregnancy-associated gastric cancer: a case report. World J Gastroenterol.2008;14(6):951-3. doi: 10.3748/wjg.14.951. 
8. Piura B. Umbilical metastasis: Sister Mary Joseph's nodule. Harefuah. 2006; 145(7):505-9, 550.

9. Abu-Hilal M, Newman JS. Sister Mary Joseph and her nodule: historical and clinical perspective. Am J Med Sci. 2009;337(4):271-3. doi: 10.1097/ MAJ.0b013e3181954187.
10. Piura B, Meirovitz M, Bayme M, Shaco-Levy R. Sister Mary Joseph's nodule originating from endometrial carcinoma incidentally detected during surgery for an umbilical hernia: a case report. Arch Gynecol Obstet. 2006;274(6):385-8. doi: 10.1007/s00404-006-0189-0. 